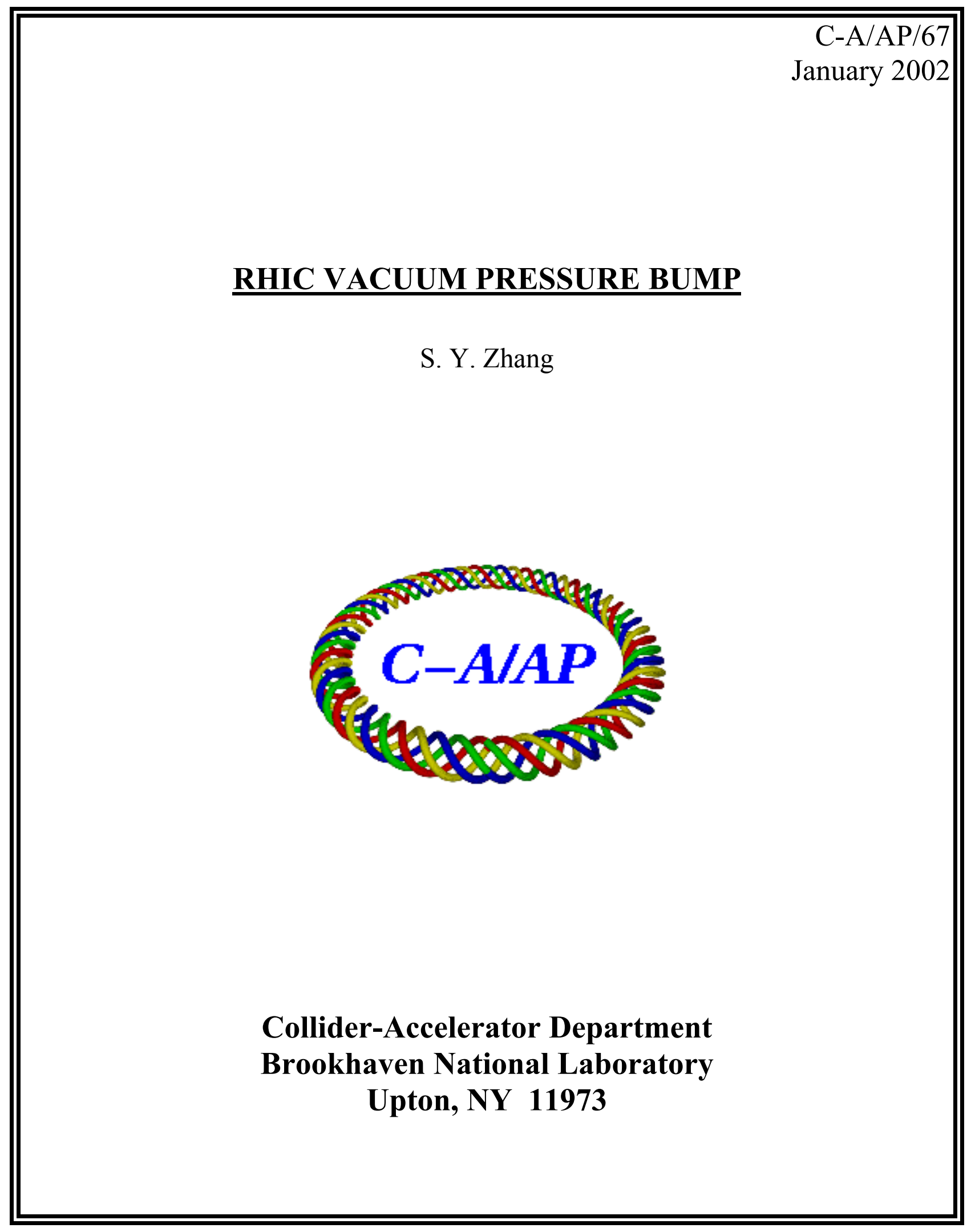




\title{
RHIC Vacuum Pressure Bump
}

\author{
S.Y. Zhang
}

January 14,2002

\begin{abstract}
Effects of the beam loss, ion and electron gas desorption on the RHIC vacuum pressure bump have been reviewed. The beam loss and ion desorption are probably not the dominant factors in the RHIC vacuum problem. On the other hand, electron desorption, given the electron multipacting, can cause serious vacuum problem. In general, the electron multipacting was not predicted for the RHIC according to the present understanding of the mechanism. However, beam loss created ions can extend electron lifetime during the bunch gap, and electron multipacting becomes possible at the RHIC. Also, the pressure rise in the RHIC Gold beam run was much worse than the usual electron cloud induced vacuum pressure rise, which was observed in many machines. One possibility is that the beam loss created ions have raised the electron density saturation limit and, therefore, contributed to further pressure rise. Some complications in diagnostics of electron multipacting by coherent tune shift in bunches will be discussed. Electron collection may indicate the coincidence between the electron multipacting and the pressure rise. It also can help to determine the electron density.
\end{abstract}

\section{Introduction}

The vacuum pressure bump has been a problem for the high intensity RHIC FY2001 gold beam run, in both 55 bunch and 110 bunch filling patterns. The pressure bump was shown up at the warm beam sections, mostly at the intersection regions. The relevant factors include the bunch intensity, bunch spacing, beam loss, and the locations.

In this note, the following issues are discussed. 
- Electron multipacting in the RHIC is, in general, not predicted by the present understanding and machine experiences of electron cloud.

- A review of several features of the RHIC vacuum pressure bump shows that the beam loss may play a role in the pressure rise.

- Beam loss, especially heavy ion beam loss, at a glancing angle can create large amount of ions and electrons. Both ions and electrons may gain energy from the passing bunches, and desorb gas from the chamber.

- Relevant issues of the ion and electron desorptions are reviewed. The ion and electron desorption rate, the energy gained from the beam and the electron density are addressed basically from the machine experience and measurements.

- The ion desorption was the source of the pressure bump in CERN ISR, and it may cause similar problem for LHC at the warm bore. However, calculation shows that it is not straightforward to explain the pressure bump at the RHIC by this mechanism. On the other hand, the ion-electron cloud generated by the beam loss can extend the secondary electron lifetime significantly, therefore, electron multipacting may happen at the RHIC. Calculation of the electron desorption induced pressure rise shows that if electron multipacting exists, it is capable to create serious vacuum problem. Moreover, the electron density may be raised beyond the space charge limit, because of the ions. Note that the pressure rise due to electron cloud has been observed in many machines, but the RHIC situation is far more serious.

- Diagnostics for electron cloud and beam loss effect is discussed. The bunch tune shift measurement has some complications. A possible electron collection using biased pick-up at some straight sections may help to determine the relevance of electrons for the pressure bump. It also helps to determine the electron density. With different bias, the pick-up can also be used to collect ions. Finally, a beam scraping study may be useful to estimate the beam loss effect. 


\section{Electron multipacting conditions}

One possible cause of the vacuum pressure rise is the beam induced electron cloud [1]. Large amount of secondary electrons may desorb significant amount of gas out of the wall. Two most critical factors in establishing electron multipacting are discussed as follows $[2,3]$.

First factor is the average energy gain of the electrons obtained during a bunch passing, which is calculated by

$$
\langle\Delta E\rangle=\frac{e}{2 m_{e}}\left(\frac{N_{b h} e Z_{0}}{2 \pi b}\right)^{2} \ln \left(\frac{b}{a}\right)
$$

where $m_{e}$ is the mass of electron, $N_{b h}$ is the charge per bunch, $Z_{0}=377 \Omega, b$ and $a$ are the radii of chamber and bunch, respectively. The peak production energy for the secondary electrons is between $300 \mathrm{eV}$ to $500 \mathrm{eV}$. If $\langle\Delta E\rangle$ is too low, then secondary yield will not be large enough for the multipacting.

Second factor for the electron multipacting is the bunch spacing, $t_{b s}$. Most secondary electrons have kinetic energy of around $3 \mathrm{eV}$. For bunch spacing at $100 \mathrm{~ns}$, these electrons travel about $10 \mathrm{~cm}$ between the bunch gap, and therefore cannot survive.

In Table 1, electron multipacting conditions in several machines are shown. Most relevant machine for the RHIC perhaps is the CERN SPS, where for LHC beam with $25 \mathrm{~ns}$ bunch spacing, the electron cloud has been observed $[1,4]$. Several electron cloud related effects, such as the vacuum pressure rise [1], beam emittance blow-up [4], beam instability [5], and the electron density [6], etc. have been extensively studied.

With the bunch spacing of $130 \mathrm{~ns}$, bunches with much higher intensity were injected at the SPS, no electron multipacting was observed [5].

Parameters with electron cloud in three lepton machines are also shown, the bunch spacing is to be noticed. The RHIC parameters are for 110 bunches with $10^{9} A u^{79+}$ per bunch, at the injection. The chamber size is at the warm bore, where vacuum pressure rise is usually observed. 


\begin{tabular}{|cccccc|}
\hline & RHIC & SPS & APS & PEPII & KEK \\
& & EC/No & & LER & LER \\
Machine radius, $R, m$ & 610 & 1100 & 175.7 & 350 & 480 \\
Kinetic energy, $E_{k}, G e V$ & 8.9 & 26 & 7 & 3.1 & 3.5 \\
Charge per bunch, $N_{b h}, 10^{10}$ & 7.9 & $6 / 25$ & 3.5 & 9 & 3.5 \\
Bunch full length, $\tau_{\ell}, n s$ & 20 & $4 / 7$ & 0.072 & 0.173 & 0.053 \\
Vertical pipe radius,,$m m$ & 61 & 22.5 & 21 & 25 & 32 \\
Beam vertical radius, $a_{v}, m m$ & 4.7 & 5.6 & 0.12 & 0.5 & 0.15 \\
Average energy gain, $\langle\Delta E\rangle, e V$ & 35 & $80 / 1394$ & 116 & 411 & 52 \\
Bunch spacing, $t_{b s}, n s$ & 106 & $25 / 130$ & 20 & 8.4 & 8 \\
\hline
\end{tabular}

Table 1: Comparison of electron multipacting conditions

Comparison of the two most critical conditions of the electron multipacting shows,

- The RHIC has the lower average electron energy gain during the bunch passing, $\langle\Delta E\rangle=35 \mathrm{eV}$, than the machines encountered electron multipacting.

- The RHIC gold beam with 110 bunches has the bunch spacing of 106 $n s$, larger than the machines with the confirmed electron multipacting. The RHIC bunch spacing is almost comparable to the SPS without electron cloud, 130 ns.

In general, therefore, present understanding of the electron multipacting predicts that the electron cloud should not happen at the RHIC for gold beam with 110 bunches.

An exception is the RHIC intersection region (IR), where two crossing beams generate irregular, but shorter, bunch spacing. Also, the chambers there have radius of about $4 \mathrm{~cm}$, giving rise to higher average energy gain $\langle\Delta E\rangle$.

In 2001 Gold beam run, however, vacuum pressure rise was observed at the warm bore of each ring with 110 bunches, and it also observed at the IRs with 55 bunches filling pattern.

In investigating the mechanism of the RHIC pressure bump, several special features of the RHIC pressure bump have been noticed. 


\section{Features of the RHIC vacuum pressure bump}

The RHIC pressure bump is different from the vacuum pressure problem encountered at other machines, for instance, the ISR due to ion desorption, SPS due to electron cloud, and LEAR due to beam loss.

Following features of the RHIC vacuum pressure bump may be helpful in understanding the RHIC situation,

- The pressure rise at the RHIC is quite unevenly distributed at the locations in the ring, even for the chambers with same or similar conditions.

- Highest pressure usually show up at the middle of the straight sections, either in a warm bore, or in an IR. This is compared with the SPS electron cloud induced pressure rise, where at the presence of dipole field, electron multipacting threshold is lower, and pressure is higher.

- Intersection region IR12 is the location where most vacuum incident happened. At IR4, the vacuum incident is rarely observed. The chamber size, length, baking condition and the material of these two IRs are the same. Only difference is that IR4 has many chamber interruptions because of the instruments, RF cavities, and other devices.

- The RHIC vacuum pressure rise is probably the most violent in comparable machines. Vacuum valve closed frequently in the Gold beam run, because the pressure increased from less than $10^{-9}$ Torr to higher than $10^{-5}$ Torr. The pressure higher than $10^{-4}$ Torr was also observed.

Note that the CERN ISR was the only machine where the beam intensity was limited by the vacuum. The mechanism is, however, understood.

Observation shows that in a few cases just a beam loss had caused the vacuum valve close. The beam loss effect, however, need to be studied for possible contributing to ion and/or electron desorption induced pressure bump, since the latter may limit the beam intensity.

\section{Beam loss effect}

\subsection{Effect of heavy ion beam loss at glancing angle}

The beam loss effect is summarized in Table 2 [7]. In general, charged particles hit the wall, secondary electrons will be generated due to the electronic 
stopping, ions (mostly positive) and neutral particles generated due to the sputtering. In Table 2, $v$ is the speed, $q$ and $A$ are the charge and mass, of the projectile.

An important factor affecting the secondary yield is the incident angle. In general, the effect is formulated as $1 / \sin \theta$, where the perpendicular incident angle is $\theta=90$ degrees. Due to difficulties in the measurement, the yield of very small incident angle is not exactly known.

\begin{tabular}{|ccc|}
\hline & Secondary Electron & Sputtering \\
Production & Electron & Ion and neutral particle \\
Mechanism & Electronic stopping & Nucl. and electr. stopping \\
Sensitive to & $v$ and $q$ & $v$ and $A, q$ for ion emission \\
$q$ factor & $q^{2}$ & $q^{3}$ for ion emission \\
Yield & $0.1 \sim 10^{6}$ & $0.1 \sim 10^{6}$ \\
$1 / \sin \theta$ factor & yes & yes \\
Emission & Peak at $3 \mathrm{eV}\left(\beta \approx 3^{-3}\right)$ & Peak at $10 \mathrm{eV}\left(\beta \approx 10^{-4}\right)$ \\
\hline
\end{tabular}

Table 2: Beam loss effect

Also, the heavy ion beam loss effect is larger than the proton beam loss. This is especially shown in the sputtering effect, where not only the secondary production is sensitive to the mass of the projectile, but also the ion production rate is proportional to $q^{3}$.

One may notice the large range of the secondary yield in Table 2, which indicates the complication of this effect. The glancing angle effect is one of the most important aspects.

\subsection{Machine observations}

The beam loss itself generates neutral particles, and also secondary ions can combine with the electrons. Beam loss induced vacuum pressure rise has been observed, especially in heavy ion machines.

- In the AGS Booster, $1 \mathrm{Mev} / u A u^{32+}$ beam loss at the injection induced transient vacuum pressure rise from $5 \times 10^{-11}$ Torr to higher than $10^{-7}$ Torr , causing significant beam loss. It is estimated that each lost $A u^{32+}$ particle generates about $10^{5}$ molecules [8]. Note that at the Booster injection, only a partial scraping is involved in the beam loss. 
- In LEAR at CERN, the vacuum pressure rise due to stored $4.2 \mathrm{Mev} / \mathrm{u}$ $\mathrm{Pb}^{54+}$ ions is effectively limiting beam intensity at the half of LHC required. It was estimated that about $5 \times 10^{5}$ molecules generated for each lost $P b^{54+}$ ion $[9,10]$.

- Recently, a dedicated study aimed at the understanding of the LEAR vacuum pressure rise was performed using LINAC3 beam of $4.2 \mathrm{Mev} / \mathrm{u}$ $\mathrm{Pb}^{53+}$ ions. It was found that at the glancing angle of $14 \mathrm{mrad}$ the molecule production was the highest, at the rate of $1.5 \times 10^{4}$ molecules per lost ion [10]. Since the test chamber length is limited, it is not very clear if the glancing angle effect has been fully explored.

Because of the difficulties in measuring the glancing incident effect for the beam loss, the effect has not been fully studied, especially for the very shallow incident angle. Moreover, almost all studies used low energy projectiles, typically in $\mathrm{keV}$ and low $\mathrm{MeV}$ region, the effect of very high energy heavy ion beam scraping on the wall is in fact unknown.

Nevertheless, it is reasonable to assume that the fully stripped $8.9 \mathrm{GeV} / \mathrm{u}$ gold ion lost at long straight sections can generate large amount of electrons, positive ions, and molecules.

In following sections, we review ion and electron desorption related parameters, then the vacuum pressure bump at the RHIC will be discussed.

\section{Ion desorption}

Ions in the chamber can be repelled to the wall by the passing bunches, hence induce gas desorption. One example is that the beam intensity of the CERN ISR was limited by the ion desorptions [11]. Also recently, interest has been shown for possible LHC warm bore pressure bump due to the ion desorption $[12,13]$.

The mechanism of the ion desorption induced pressure bump is described as follows.

Positive ions can be generated either by the beam ionization with the residual gas, or by the beam loss. It is shown in Table 2 that the sputtering generated ions are mostly at the energy of $10 \mathrm{eV}, \beta \approx 10^{-4}$, the ionization generated ion energy is about the same. Therefore, these ions move slowly. Comparing with the secondary electron motion during $100 \mathrm{~ns}$ bunch spacing, the equivalent one for these ions is $3 \mu \mathrm{s}$. In fact, the ion lifetime could be 
longer, given the co-existence of electrons. These ions will be repelled during many bunches passing, until hitting the wall.

The key factors for the possible ion desorption induced pressure bump are as follows,

- The ion desorption rate, which is much larger than the electron desorption rate.

- Ion energy gained from the passing beam, which depends not only on the beam intensity, but also on the ion lifetime.

- The generation of the ions.

The first two factors will be discussed in this section, and the ion generation will be discussed together with the ion desorption induced pressure bump.

\subsection{Ion desorption rate}

For unbaked and baked stainless steel surface, the ion desorption rate is shown in Table 3, [14]

\begin{tabular}{|ccccc|}
\hline Projectile kinetic energy, $k e V$ & 0.5 & 1 & 2 & 3 \\
Yield, unbaked surface & 4.1 & 5.5 & 7.1 & 7.6 \\
Yield, baked surface & 0.7 & 0.9 & 1.2 & 1.3 \\
\hline
\end{tabular}

Table 3: Ion desorption rate

The data were obtained using $N_{2}^{15+}$ ions as the projectile, and the yield is only for $\mathrm{H}_{2}$ molecules. The yields of $\mathrm{CO}, \mathrm{CO}_{2}, \mathrm{CH}_{4}$ are $78 \%, 33 \%$ and $8 \%$ of $H_{2}$, so the total yield will be larger by more than factor of 2 .

The yield rapidly increases for projectile energy increasing, up to $0.5 \mathrm{keV}$, then saturates at around $1 \mathrm{keV}$. The ratio of the yield with $2 \mathrm{keV}$ projectile for unbaked and baked surface is $7.1 / 1.2=5.9$, however, in [15], it is only $9 / 4=2.5$.

Compared with the electron desorption rate not larger than 0.1 , the ion desorption rate is much larger. 


\subsection{Ion energy gain from passing beam}

Ions will receive kicks from many passing bunches, until hitting the wall. Therefore, the beam potential is used as the criterion for the ion energy gain, which is [16],

$$
V_{\text {pot }}=\frac{I Z_{0}}{2 \pi}\left(0.5+\ln \left(\frac{b}{a}\right)\right)
$$

where $I$ is the average beam current.

In the case of ISR, a DC beam with $10^{14}$ protons has a potential of 700 $V$. The vacuum pressure bump was explained that with the ion desorption rate of 4 , the ionization generated ions were repelled by the proton beam to the wall, and generate more gas than the pumps can handle.

For bunched beams, using the peak current and squeezed beam size at IR of the LHC, beam potential is very high, therefore, possible vacuum bump at the warm sections due to ion desorption is currently under study $[12,13,15,16]$.

Limited ion lifetime, however, raises a question that how accurate to equal the ion energy to the beam potential, especially for using the potential with peak current. Further studies are certainly of interest.

In Table 4, the comparison of the beam potential with average and peak current is shown for the RHIC, ISR, and LHC.

\begin{tabular}{|cccc|}
\hline & RHIC & ISR & LHC \\
Machine radius, $R, m$ & 610 & 150 & 4243 \\
Kinetic rnergy, $E_{k}, G e V$ & 8.9 & 26 & 450 \\
Charge per bunch, $N_{b h}, 10^{10}$ & 7.9 & 10000 & 11 \\
Vertical pipe radius, $b, m m$ & 61 & 30 & 18 \\
Vertical beam radius, $a, m m$ & 4.7 & 5 & 0.28 \\
Average beam current, $I, A$ & 0.11 & 5.1 & 0.56 \\
Beam potential, $V_{\text {pot. }}, V$ & 20 & 700 & 156 \\
Peak beam current, $I_{\text {peak }}, A$ & 1 & 5.1 & 28 \\
Beam potential, $V_{\text {pot.peak }}, V$ & 184 & 700 & 7835 \\
\hline
\end{tabular}

Table 4: Comparison of beam potential

We may see that for RHIC, even using the peak beam current, the potential is no more than $200 \mathrm{~V}$. In calculating the ion desorption induced pressure bump, we therefore take the ion desorption rate $\eta=1$ for the RHIC. 


\section{Electron desorption}

Since the electron desorption rate is not large, only large amount of electrons generated from the electron multipacting can cause serious problem for vacuum pressure. For possible electron multipacting at the RHIC, the two factors discussed in Section II have to be reconsidered, because,

- With certain amount of beam loss in a glancing angle, secondary ions and electrons are generated and an ion-electron cloud is formed. Electrons will stay for longer time than the one determined by the electron motion without ions. Therefore, the bunch spacing for RHIC 110 bunches, $t_{b s}=106 \mathrm{~ns}$, may allow electron multipacting.

- A new study shows that with the elastic scattering, the low energy electron generated secondary yield is higher than the one predicted by the Seiler model $[17,18]$. This implies that the average electron energy gain $\langle\Delta E\rangle=35 \mathrm{eV}$ of RHIC, shown in Table 1, may not be used to rule out the possibility of electron multipacting.

The electron desorption rate and the electron density are two important factors for possible electron multipacting induced pressure bump.

\subsection{Electron desorption rate}

The electron desorption rate has been studied in [19], the result is shown in Table 5 .

\begin{tabular}{|cccccccc|}
\hline Electron kin. energy, $e V$ & 20 & 50 & 100 & 200 & 500 & 1000 & 1600 \\
Yield, unbaked surface & 0.005 & 0.011 & 0.02 & 0.032 & 0.046 & 0.054 & 0.058 \\
\hline
\end{tabular}

Table 5: Electron desorption rate

The yield components are $40 \% \mathrm{H}_{2}, 40 \% \mathrm{CO}$, and $20 \%$ water. It can be seen that the yield increases for the projectile electron energy raising up to $500 \mathrm{eV}$, then flattened.

In recent reports, an electron desorption rate of 0.1 is frequently used $[4,20]$, which will be taken for a quick estimate of the electron desorption effect. 


\subsection{Electron density}

Electron density is an important factor for electron desorption induced pressure rise. The CERN SPS and PS measurement data in electron cloud studies will be used as the reference.

With the electron cloud build-up, a pick-up in SPS collected around $5 \times$ $10^{8}$ electrons per batch with 72 bunches at the bunch intensity $N_{b h}=5 \times 10^{10}$ [6]. Pick-up has a triangle shape of the dimension $12.5 \mathrm{~cm} \times 15 \mathrm{~cm}$. Effective length of pick-up is about $10 \mathrm{~cm}$. The electron multipacting started at about 35 bunches in the bunch train, therefore, the electron line density is $\lambda_{e}=$ $5 \times 10^{8} / 0.1 / 35=1.43 \times 10^{8} \mathrm{~m}^{-1}$. The LHC beam bunch length is $1.2 \mathrm{~m}$, the neutralization factor is, therefore, $\eta_{e}=1.2 \lambda_{e} / N_{b h}=1.2 \times 1.43 \times 10^{8} / 5 \times 10^{10}=$ 0.0034 .

This line density is agreeable with the one observed at the PS, where a pick-up at the PS extraction transfer line collected $10^{9}$ electrons per LHC batch, at the bunch intensity of $N_{b h}=11 \times 10^{10}$ [21].

A serious question is the transverse size of the electron cloud. Assuming the electrons uniformly distributed with the SPS proton beam with $a_{v}=5.6$ $\mathrm{mm}$ and $a_{h}=7.3 \mathrm{~mm}$, the electron density is $\rho_{e}=\lambda_{e} / \pi a_{v} a_{h}=1.43 \times$ $10^{8} /(\pi \times 0.0056 \times 0.0073)=1.1 \times 10^{12} \mathrm{~m}^{-3}$. Assuming the electrons uniformly distributed within the chamber of $b_{v}=2.25 \mathrm{~cm}$ and $b_{h}=7 \mathrm{~cm}$, the electron density is much smaller, $\rho_{e}=2.9 \times 10^{10} \mathrm{~m}^{-3}$. This issue is important in determining the electron induced coherent tune shift, it is also important in terms of beam emittance growth and instabilities.

For electron desorption effect, fortunately, the neutralization factor is sufficient in the estimating the pressure rise.

The neutralization factor of the RHIC at above electron multipacting threshold is assumed to be $\eta_{e}=0.01$.

\section{$7 \quad$ Vacuum pressure bump}

Vacuum pressure rises when either ion or electron desorbed gas increases to more than the vacuum pump can handle. In the following, we discuss the possible RHIC pressure bump due to ion and electron desorption. The experimental parameters in CERN ISR and SPS studies will be used.

It is useful to first take a look at the beam loss induced pressure rise, which was happened in RHIC Gold beam operation, sometimes caused the 
vacuum valve close.

\subsection{Beam loss induced pressure bump}

Take an intersection region as an example. Let the IR length be $\ell_{I R}=20$ $m$, with average chamber radius of $b=5 \mathrm{~cm}$, the total volume is $V_{I R}=0.16$ $m^{3}$. The molecule density at room temperature is $k_{m}=3.3 \times 10^{22} \mathrm{~m}^{-3}$ for $P=1$ Torr. For the pressure of $P=10^{-9}$ Torr, there are total $k_{m} P V_{I R}=$ $3.3 \times 10^{22} \times 10^{-9} \times 0.16=5.3 \times 10^{12}$ molecules in the chamber.

The RHIC vacuum pumping speed is $S \approx 25 \mathrm{\ell s}^{-1} \mathrm{~m}^{-1}$ at the warm beam sections [22], which implies that at the pressure of $P=10^{-9}$ Torr, the molecules pumped out are $k_{m} P S \ell_{I R}=3.3 \times 10^{22} \times 10^{-9} \times 25 / 1000 \times 20=$ $1.65 \times 10^{13} \mathrm{~s}^{-1}$. The thermal outgassing is assumed to be the same as for the pressure balance.

For a beam loss of $10^{7}$ ions per second in the IR, taking the yield as $5 \times 10^{5}$ molecules per lost ion, total $5 \times 10^{12}$ molecules are generated per second. Without pumping, the pressure is doubled to $P=2 \times 10^{-9}$ Torr in a second.

Considering pumping with constant speed, the balanced pressure at $P=$ $2 \times 10^{-9}$ Torr requires $1.65 \times 10^{13}$ molecules to be created every second. This number is 3 times larger the one needed to double the vacuum pressure without pumping, $5.3 \times 10^{12}$. Therefore, the beam loss rate should be about $3 \times 10^{7}$ per second with the pumping. Since higher pressure requires even larger beam loss, it is unlikely that this mechanism is a dominant one in creating RHIC pressure rise that caused valve close.

On the other hand, a large beam loss at a short time can cause valve close. Taking the yield rate of $5 \times 10^{5}$, a beam loss of $10^{9}$ ions per second in an IR can generate $5 \times 10^{14}$ molecules, which is enough to raise the pressure to $P=10^{-7}$ Torr. For the same beam loss happened in a shorter time, the transient pressure rise may go higher, partially clogging the vacuum pump. This kind of pressure rise has been observed, for instance, in Ramp1754. The real vacuum pressure rise due to the beam loss was sometimes higher than $10^{-4}$ Torr. 


\subsection{Ion induced pressure bump}

For the ion desorption induced pressure rise, the mechanism can be described as $[11]$

$$
P=\frac{Q_{0}}{S-\eta \sigma I / e}
$$

where $P$ is the pressure in Torr, $Q_{0}$ is the thermal outgassing, $S$ is the pumping speed in $\ell s^{-1} m^{-1}, \eta$ is the ion desorption rate, $\sigma$ is the gas ionization cross section, $I$ is the average beam current. The mechanism is that the ions are produced by the beam ionization with the residual gas, mainly $C O$, then these ions are repelled to the wall by the passing beam. When the beam intensity is above the threshold, the gas desorption rate is larger than the pumping rate, the pressure bump is produced.

The ion desorption rate with the ion energy of $700 \mathrm{eV}$ at the ISR is assumed to be $\eta=4$, see Tables 3 and 4 . Taking the ionization cross section of $C O$ at $26 \mathrm{GeV}$ as $\sigma=10^{-22} \mathrm{~m}^{2}$, for beam current of $5.1 \mathrm{~A}$, the pump speed of $S \geq \eta \sigma I / e=13 \mathrm{ls}^{-1} \mathrm{~m}^{-1}$ is required to prevent the pressure bump. This pumping speed is close to the initial capacity of the ISR vacuum system. The ISR pumping was, therefore, improved in a period of years, the beam intensity was increased to about $10^{15}$ protons per ring.

Consider the RHIC with the same mechanism, for $I=0.11 \mathrm{~A}$, taking $\eta=1$, required pump speed is only $S=0.07 \ell s^{-1} m^{-1}$, which is far less than the RHIC capability of $S \approx 25 \mathrm{ls}^{-1} \mathrm{~m}^{-1}$ at the warm sections.

Take look in other way. For the same IR, using $I=0.11 A, \eta=1$, and $\sigma=10^{-22} \mathrm{~m}^{2}$, the ionization produced ions create total $k_{m} P \eta \sigma I / e \ell_{I R}=$ $3.3 \times 10^{22} \times 10^{-9} \times 10^{-22} \times 0.11 / e \times 20=4.5 \times 10^{10} \mathrm{~s}^{-1}$ molecules in this region, which is not significant enough in playing a major role in the RHIC pressure bump.

\subsection{Electron induced pressure bump}

Using the measurement data obtained in the SPS for the electron cloud caused pressure rise, the RHIC pressure bump will be analyzed.

Taking the SPS electron collected as $10^{9}$ per batch per pick-up [6,21], and the average length of the pick-up as $10 \mathrm{~cm}$, which gives rise to $10^{10}$ electrons per batch-meter. Taking the electron desorption rate of 0.1 , and the revolution frequency of $f_{0}=43 \mathrm{kHz}$, the electron generated molecules are $0.1 \times 10^{10} f_{0}=4.3 \times 10^{13} s^{-1} m^{-1}$. For the pressure $P=10^{-7}$ Torr , 
pumping speed of $S=1 \ell s^{-1} m^{-1}$ can handle the molecules of $k_{m} P S=$ $3.3 \times 10^{22} \times 10^{-7} / 10^{3}=3.3 \times 10^{12} s^{-1} m^{-1}$. Therefore, the SPS electron created vacuum pressure is balanced at $S=4.3 \times 10^{13} / 3.3 \times 10^{12}=13$ $\ell s^{-1} m^{-1}$ at $P=10^{-7}$ Torr. The pumping speed and the vacuum pressure are approximately agreeable with the SPS parameter and observation.

For RHIC gold beam with $10^{9}$ ions per bunch, the bunch charge is $N_{b h}=$ $7.9 \times 10^{10}$. The bunch length is $6 \mathrm{~m}$ at the injection, therefore, the bunch charge line density is $1.32 \times 10^{10} \mathrm{~m}^{-1}$. With the neutralization factor of $\eta_{e}=0.01$, electron line density is $\lambda_{e}=1.32 \times 10^{8} \mathrm{~m}^{-1}$. Assuming half of the 110 bunches have developed electron cloud, with the revolution frequency of $f_{0}=78 \mathrm{kHz}$, and the electron desorption rate of 0.1 , the generated molecules are $\lambda_{e} f_{0} \times 110 / 2 \times 0.1=5.66 \times 10^{13} \mathrm{~s}^{-1} \mathrm{~m}^{-1}$. Therefore, the equivalent pump speed with the electron desorbed gas production is $S=5.66 \times 10^{13} / 3.3 \times$ $10^{12}=17.2 \ell \mathrm{s}^{-1} \mathrm{~m}^{-1}$, which is not far away from $25 \ell \mathrm{s}^{-1} \mathrm{~m}^{-1}$.

Several issues need to be discussed.

- If the pumping speed is indeed $S=17.2 \mathrm{\ell s}^{-1} \mathrm{~m}^{-1}$, then the vacuum pressure will rapidly rise to $P=10^{-7}$ Torr, and stay at this level. At higher pressure, say $P=10^{-6}$ Torr, the pumping efficiency will drop probably by factor of 2 [22], however, the molecules that pumped out are still 5 time of that at $P=10^{-7}$ Torr. Therefore, the molecule yield higher than the one described above is needed to explain the observed pressure.

- It is possible that the electron desorption rate is higher, and/or the real pumping speed is lower, however, the electron density is a more important factor. Usually, the electron density is limited by the space charge [3], however, this limit is removed if there exist large amount of ions. It is possible that the electron density is higher than the estimated. Note that the vacuum pressure rise was observed in almost all machines having electron cloud, but there the pressure rises are more evenly distributed in the ring, more gentle, and no valve close, or rises to $P \geq 10^{-5}$ Torr were reported.

- Because of the same reason, the secondary electrons may survive for the bunch-train gap, which is about $1.2 \mu \mathrm{s}$ for both 55 bunch and 110 bunch filling patterns. In this case, electron multipacting will take place on all the bunches, continuously. Threshold will be lower, effect on the vacuum pressure will be larger. 


\section{Diagnostics}

Diagnostics is needed to determine the cause of the RHIC vacuum pressure bump. The coherent tune shift for the bunches in the train may be used to detect the electron cloud. Some complications, however, may make this approach less reliable. A better way to detect the electron cloud maybe the use of electron collectors placed at the intersection region or at the warm bore. If there is coincidence between the electrons and the vacuum pressure rise, then the cause of the vacuum problem can be determined. Also, the local electron density can be determined, which as discussed above, is an important factor in the pressure rise. The third study can be observing the vacuum pressure variation by scraping beams on the wall.

In the following, we have a little more detailed discussion for each of these diagnoses.

\subsection{Coherent tune shift due to electrons}

The coherent tune shift due to the electron cloud is calculated using $[4,20]$,

$$
\Delta Q_{v, h}=\frac{r_{p} C \bar{\beta} \rho_{e} b_{h, v}}{\gamma\left(b_{h}+b_{v}\right)}
$$

where $r_{p}=1.535 \times 10^{-18} \mathrm{~m}$ is the classical radius of proton, $C$ is the machine circumference, $\bar{\beta}$ is the average beta function, $b_{h}$ and $b_{v}$ are horizontal and vertical chamber radii, and $\rho_{e}$ is the electron density.

Applying this formulation to the SPS, where $C=6,910 \mathrm{~m}, \bar{\beta}=40 \mathrm{~m}$, $\gamma=28.7, b_{h}=7 \mathrm{~cm}, b_{v}=22.5 \mathrm{~cm}$, and the electron density $\rho_{e}$ is assumed to be $10^{12} \mathrm{~m}^{-3}$, we get $\Delta Q_{v}=0.011$ and $\Delta Q_{h}=0.004$. About 0.01 in vertical tune shift observed in the measurement, but no appreciable shift detected in horizontal, which is not understood yet [24].

The mechanism of the coherent tune shift is explained [20] by the electric force the bunch encountered when it passes through the static electron cloud of uniform density $\rho_{e}$ within the chamber. Question exists for using the electron density of $\rho_{e}=10^{12} \mathrm{~m}^{-3}$, since the pick-up collected electrons [4,21] agree with this density only if the electron cloud has the same size as the beam. For electrons uniformly distributed in the chamber [20], the density is only $\rho_{e}=2.9 \times 10^{10} \mathrm{~m}^{-3}$.

To estimate the RHIC beam tune shift due to electrons, a factor of $q / A=$ $79 / 197=0.4$ should be applied. Using $C=3,833 m, \bar{\beta}=22 m, \gamma=10.5$, 
$b_{h}=b_{v}=5 \mathrm{~cm}$, and the electron density $\rho_{e}$ is also assumed to be $10^{12} \mathrm{~m}^{-3}$, one gets $\Delta Q=0.0024$ for both horizontal and vertical.

Some comments are due for this measurement.

- To be accurate, the factor $C$ in the formulation should be replaced by the effective length, $L$, of the electron cloud in the ring. If the electron cloud did happened in the RHIC, it does not happen in the whole ring. Therefore, it is needed to determine $L$.

- As discussed above, the electron density is a big uncertain factor. One may speculate, however, because of the ions, the electron density can be larger than the assumed one, or the neutralization factor $\eta_{e}>0.01$. This may offset the factor of $L<C$ in calculating the tune shift.

- Also as discussed above, the bunch-train gap in the ring might not be long enough to dump the electrons, hence there is a possibility that the electron multipacting takes place continuously for all circulating bunches. In this case, the shift will take place for all the bunches, along with the time.

- The electron motion within the heavy ion beam is expected to be violent. Also, the ion-electron cloud effect needs to be considered.

- Without electron multipacting, the electrons exist in the ring, for instance, from beam loss. These electrons can contribute to the tune shift.

\subsection{Electron collection}

The electron collection using a pick-up may provide more information.

- Coincidence between the electron multipacting and the vacuum pressure rise can help to determine the cause of the pressure problem.

- The electron line density can be analyzed from the collected electrons. A comparison between this density and the observed pressure rise can provide valuable information in understanding the pressure rise.

- By changing collector bias, ions can also be collected. 


\subsection{Beam loss effect}

The effect of high energy heavy ion beam loss, especially at glancing angle, is not clearly understood. A study will be helpful for better evaluation of the effect.

The beam can be steering at an IR, the vacuum pressure, the beam loss, and the gas component analysis using PPA can be performed.

\section{Acknowledgment}

I like to thank H.C. Hseuh, D. Trbojevic, W. MacKay and T. Roser for helpful discussions.

\section{References}

[1] M. Jemeniz, "SPS Vacuum Observations and Electron Scrubbing with LHC Beams", Chamonix XI, pp.160-162, CERN, 2001.

[2] O. Grobner, "Beam Induced Multipacting", EPAC 98, pp.3589-3591, Stockholm, Sweden,1998.

[3] L. Vos, "Electron Cloud: an Analytic View", LHC Project Note, 150, CERN, July 1998.

[4] F. Zimmermann, "Electron Cloud Simulations: An Update', Chamonix XI, pp.144-159, CERN, 2001.

[5] G. Auduini, "Observation of the Electron Cloud Effects at the CERN SPS", 8th ICFA Workshop, Santa Fe, New Mexico, Feb. 2000.

[6] W. Hofle, "Observations of the Electron Cloud Effect on Pick-up Signals in the SPS", Chamonix X, pp.112-118, CERN, 2000.

[7] "Sputtering by Particle Bombardment, I", Edited by R. Behrisch, Springer-Verlag, New York, 1981.

[8] S.Y. Zhang and L.A. Ahrens, "Gold Beam Losses at the AGS Booster Injection", Proc. 1999 PAC, pp.3294-3296, New York, 1999. 
[9] J. Bosser et. al., "Results on Lead Ion Accumulation in LEAR for the LHC", EPAC98, pp. 253-255, Stockholm, Sweden,1998.

[10] J Hansen, et al. "Results from First Experiment to Measure Lead Ion Induced Desorption at LINAC3", LHC/VAC Note, 2001-007, CERN, July, 2001.

[11] O. Grobner, "Dynamic outgassing", CERN 99-05, pp.127-138, 1999.

[12] O.B. Malyshev, "The Ion Impact Energy on the LHC Vacuum Chamber Walls", EPAC 2000, pp. 951-953, Vienna, Austria, 2000.

[13] I. Collins, et. al., "Vacuum Calculation for the LHC Experimental Beam Chambers", PAC2001, Chicago, 2001.

[14] I.R. Collins, et al, "Vacuum Stability for Ion Induced Gas Desorption", Int. Workshop on Performance Improvement of $e^{+} e^{-}$Factories, Sep. 1999, KEK, Japan.

[15] I.R. Collins, et al, "Mechanical and Vacuum Stability Design Criteria for the LHC Experimental Vacuum Chambers", EPAC98, pp.2202-2205, Stockholm, Sweden,1998.

[16] W.C. Turner, "Ion Desorption Stability in Superconducting High Energy Physics Proton Colliders", J. Vac. Sci. Technol., A14 (4), pp. 2026-2038, July/Aug 1996.

[17] H. Seiler, "Secondary Electron Emission in the Scanning Electron Microscope", J. Appl. Phys. 54, R1, 1983.

[18] N. Hilleret, "The Secondary Electron Yield of Copper: New Experimental Results and their Implications", Two-Stream Workshop, KEK, Japan, Sep. 2001.

[19] R.S. Calder et. al., "Electron Induced Desorption from AVESTA SKRN Stainless Steel", CERN-ISR-VA/72-34, 1972.

[20] F. Zimmermann, "The Electron Cloud Instability: Summary of Measurements and Understanding", PAC2001, Chicago, 2001.

[21] R. Cappi et. al. "Electron Cloud Effects in the CERN PS", PAC2001, Chicago, 2001. 
[22] H.C. Hseuh, private communication.

[23] Y. Baconnier et. al., "Neutralization of Accelerator Beams by Ionisation of the Residual Gas", CERN 94-01, pp. 525-561, 1994.

[24] G. Arduini, et al, "Transverse Behavior of the LHC Proton Beam in the SPS: An Update", PAC2001, Chicago, 2001. 